(6) OPEN ACCESS

\title{
Intergenerational social mobility and leisure-time physical activity in adulthood: a systematic review
}

\author{
Ahmed Elhakeem, ${ }^{1}$ Rebecca Hardy, ${ }^{1}$ David Bann, ${ }^{2}$ Rishi Caleyachetty, ${ }^{1}$ \\ Theodore D Cosco, ${ }^{1}$ Richard PG Hayhoe, ${ }^{3}$ Stella G Muthuri, ${ }^{1}$ Rebecca Wilson, ${ }^{1}$ \\ Rachel Cooper ${ }^{1}$
}

\begin{abstract}
- Additional material is published online only. To view please visit the journal online (http://dx.doi.org/10.1136/ jech-2016-208052).
\end{abstract}

${ }^{1} \mathrm{MRC}$ Unit for Lifelong Health and Ageing at UCL, London, UK ${ }^{2}$ Centre for Longitudinal Studies, UCL Institute of Education, London, UK

${ }^{3}$ Department of Population Health and Primary Care, Norwich Medical School University of East Anglia, Norwich, UK

\section{Correspondence to} Ahmed Elhakeem, MRC Unit for Lifelong Health and Ageing at UCL, 33 Bedford Place, London WC1B 5JU, UK;

ahmed.elhakeem.13@ucl.ac.uk

Received 8 July 2016 Revised 3 November 2016 Accepted 24 November 2016 Published Online First 15 December 2016

\section{ABSTRACT}

Aim To systematically review the association between intergenerational social mobility and leisure-time physical activity (LTPA) in adulthood, in order to assess all published evidence relating to the hypothesis that adults socially mobile between childhood and adulthood will have different levels of LTPA than those in the same socioeconomic group across life.

Methods A systematic review was carried out following the Preferred Reporting Items for Systematic Reviews and Meta-Analyses (PRISMA) guidelines. Studies were identified by searching databases (MEDLINE, Embase, PsycINFO) and reference lists. Eligible studies examined associations between any indicator of social mobility, based on at least one measure of parental socioeconomic position (SEP) and one measure of own adult SEP, and LTPA in adulthood.

Results 13 studies comprising a total of 44000 participants from the UK, Finland, Sweden, Australia, USA and Brazil were included. Participants were aged 16-70 years and were from population-based surveys, occupational cohorts and primary care registries. Most studies $(n=9)$ used occupational class measures to identify social mobility; education $(n=4)$ and income $(n=1)$ were also used. There was consistent evidence in nine of the 13 studies that stable high socioeconomic groups tended to report the highest levels of participation in LTPA and stable low socioeconomic groups the lowest. Upward and downwardly mobile groups participated in LTPA at levels between these stable groups.

Conclusions Cumulative exposure to higher SEP in childhood and adulthood was associated with higher LTPA in adulthood. Thus, a potential outcome of policies and interventions which aim to minimise exposure to socioeconomic adversity may be increased LTPA among adults.

Trial registration number CRD42016036538.

\section{BACKGROUND}

Regular physical activity improves physical health and mental well-being and reduces risk of chronic diseases including cardiovascular disease. ${ }^{1-3}$ It is therefore important to understand the factors operating across life which may influence participation. Reviews of cross-sectional studies have reported that adults ${ }^{4-6}$ from lower socioeconomic groups tend to participate less frequently in leisure-time physical activity (LTPA) when compared with their more advantaged peers. Systematic reviews of longitudinal studies ${ }^{7} 8$ have subsequently concluded that, despite heterogeneity in findings, lower socioeconomic position (SEP) in childhood tended to be associated with less LTPA in adulthood, and that associations were partly explained by adjustment for adult SEP.

Intergenerational social mobility, that is, changes in the level of SEP of offspring in adulthood when compared with their parents', may also be related to LTPA during adult life. Several alternative hypotheses have been proposed to explain how changes in SEP across life may relate to LTPA in adulthood. For example, adult LTPA may be predominantly influenced by socialisation effects of childhood SEP (origins hypothesis) or mostly by those of current SEP (destination hypothesis). ${ }^{9}$ Alternatively, under the maximisation hypothesis, ${ }^{10}$ those experiencing upward social mobility may adopt LTPA levels found in their destination SEP while the downwardly mobile may retain LTPA rates found in their SEP of origin. Accumulation of additive effects whereby higher childhood and adulthood SEP increase probability of participating in LTPA in a cumulative fashion is also possible, ${ }^{11}$ as is effect modification by adult SEP (synergistic/antagonistic effects). ${ }^{11}$

Studies of other cardiovascular disease risk factors generally find evidence of cumulative additive effects. ${ }^{12-16}$ For example, socially mobile study participants from New Zealand had levels of cardiorespiratory fitness at age 26 in-between the socially stable groups. ${ }^{12}$ Lower SEP also tends to cumulatively increase subsequent risks of overweight and obesity. ${ }^{13-15}$ These findings suggest that adults who have a different level of SEP to their parents might have a different probability of participation in LTPA than others whose SEP remains stable between childhood and adulthood. Therefore, a systematic review was carried out to assess all published evidence relating to the hypothesis that adults socially mobile between childhood and adulthood will have different levels of LTPA when compared with those in the same socioeconomic group across life.

\section{METHODS}

This systematic review was carried out in accordance with the Preferred Reporting Items for Systematic Reviews and Meta-Analyses (PRISMA) guidelines ${ }^{17}$ and the protocol was registered with The International Prospective Register of Systematic Reviews (PROSPERO) (registration number: CRD42016036538).

\section{Eligibility criteria}

Prospective and retrospective cohort studies published in English examining the association between changes in SEP from childhood to adulthood (ie, 
intergenerational social mobility) and LTPA in adulthood were included. Eligible measures of intergenerational social mobility were those derived based on at least one measure of childhood SEP ( $\leq 18$ years) and one comparable measure of adult SEP, ${ }^{9}$ with SEP representing any resource and/or prestige-based measures of position within a societal structure (eg, occupational class, income). ${ }^{18}{ }^{19}$ Any LTPA outcome ${ }^{20}$ recorded at or after assessment of adult SEP was eligible for inclusion. Excluded were studies with non-LTPA outcomes (eg, exclusively workrelated physical activity), LTPA measured before adult SEP and studies of institutionalised participants (eg, care home residents).

\section{Search strategy and study selection}

Embase (from 1980), MEDLINE (from 1946) and PsycINFO (from 1806) were searched up to October 2015 using keywords (see online supplementary file S1). Duplicates were removed using OvidSP and Endnote. Two independently working reviewers (from AE, RCa, TDC, RPGH, SGM and RW) carried out initial title and abstract screening (to exclude papers that were definitely ineligible) followed by a detailed full-text screening of remaining papers (to exclude papers not meeting all inclusion criteria, with reasons for exclusion recorded). Reference lists of included papers were searched to identify any other eligible studies (figure 1). Any disagreements between reviewers were resolved through discussion and consultation with RCo and RH.

\section{Data extraction and quality assessment}

Data extraction and quality assessment of each included paper were carried out by two independently working reviewers using a standardised data extraction form (similar to that developed for our previous review) ${ }^{721}$ and a modified Newcastle-Ottawa Quality Assessment Scale ${ }^{22}$ that was developed specifically for this review (see online supplementary file S2). Extracted items were study details (including design, setting and sample size), exposure and outcome details (including how social mobility and LTPA were derived and when these were ascertained), age, sex and birth years of included participants, statistical methods used including adjustment for covariates and lists of potentially eligible papers in reference lists (see online supplementary file S2). All data relating to the association of interest were extracted. Owing to heterogeneity in methods of assessment and analysis between studies, a meta-analysis was not deemed to be appropriate. Quality was judged based on representativeness of the study and source populations, adjustment for covariates, length of follow-up, whether childhood SEP was prospectively or retrospectively ascertained and methods used to assess LTPA. Quality scores were based on the average of two reviewers' ratings (possible values from lowest (0) to highest (9) quality rating).

\section{RESULTS}

Of 1199 unique citations retrieved from database searches, 13 studies reported in 17 publications ${ }^{23-39}$ were eligible for inclusion in the review (figure 1).

\section{Characteristics of included studies}

Study characteristics are summarised in table 1 . Six were from the UK, ${ }^{23-31}$ two from Finland, ${ }^{32} 33$ one from Sweden, ${ }^{34}$ two from the USA ${ }^{35} 36$ and one study each from Australia ${ }^{37} 38$ and Brazil. ${ }^{39}$ Study participants were mostly from population-based surveys. Others were sampled from primary care registries ${ }^{24-27}$ and three occupational cohorts: 27 workplaces in West of Scotland, ${ }^{30} 31$ and male ${ }^{35}$ and female ${ }^{36}$ physicians from the USA. Age at LTPA assessment ranged from late adolescence to old age with the majority of study participants aged 30 and older (table 1). Birth years were between 1900s and 1980s.

Parental SEP was prospectively ascertained in five studies (table 1). Changes in occupational class from parent to adult offspring was the most commonly used indicator of social mobility (9/13); educational attainment of parents and their offspring were compared in four studies while income mobility was studied only in a Pelotas birth cohort, Brazil. ${ }^{39}$ Participants were usually classified into four groups depending on whether they were socially mobile upwards/downwards or stable between childhood and adulthood. Four studies present results across more than four mobility groups. ${ }^{28} 30333738$ Physicians $^{35} 36$ were compared by childhood SEP (ie, implying stable high and upward social mobility). In all studies, LTPA was assessed through self-completed questionnaire or at face-to-face interview and two studies present outcomes combining work-related activity and LTPA. ${ }^{23}{ }^{29}$ Five studies had low quality rating (range $=1-2.5$ ) and four were of medium-to-high quality (range $=6-7)($ table 1$)$.

\section{Results of included studies}

Most studies present estimates of LTPA as prevalence across stable and mobile socioeconomic groups (table 2). Statistical models were used in some studies and these were either unadjusted or adjusted for age and/or sex. Popham ${ }^{28}$ examined age and sex-adjusted associations with alternating adjustment

Figure 1 Systematic review flow chart.

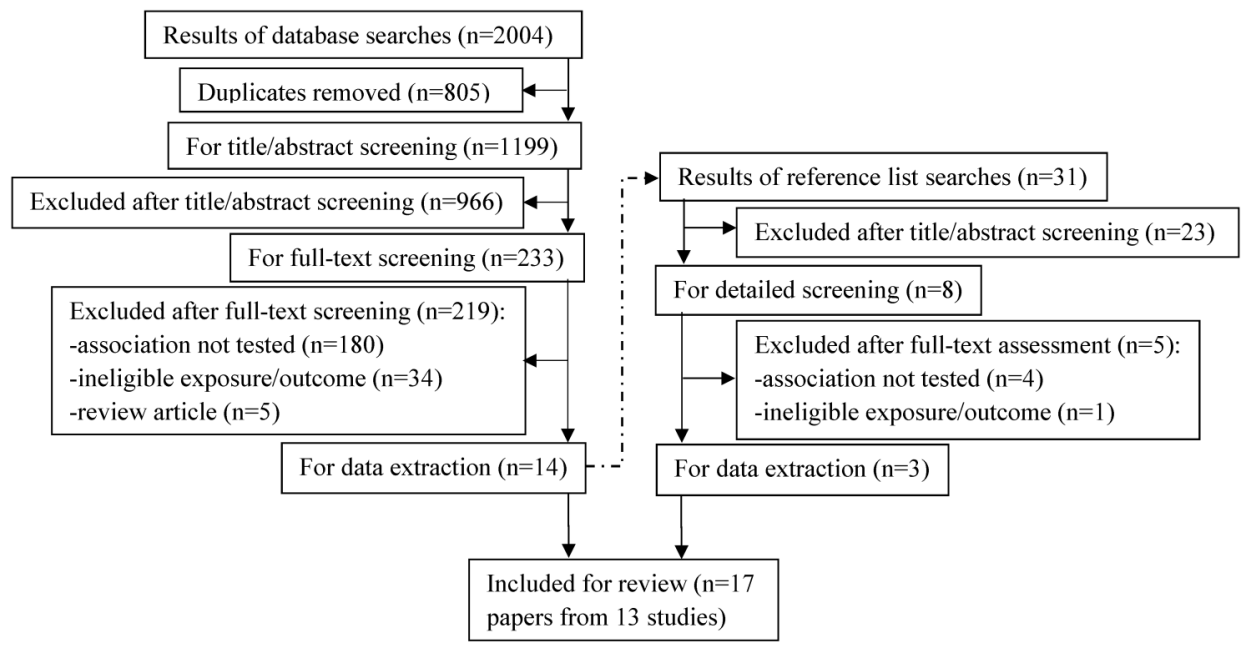


for childhood and adulthood SEP while findings from Brazil were adjusted for skin colour. ${ }^{39}$ Five studies present separate results for men and women (table 2). Nine of the 13 studies presented some evidence of associations of intergenerational mobility and stability of SEP with LTPA. Results are summarised in table 2 and the following paragraphs.

In men and women from the 1946 British birth cohort (Medical Research Council (MRC) National Survey of Health and Development), ${ }^{23}$ previously derived latent classes of physical activity types reported between ages 36 and $53^{40}$ were associated with occupational and educational mobility and stability from parent to offspring (table 2). The highest prevalence of sports and other LTPA was found among those remaining in non-manual occupations (and high educational groups) whereas the lowest prevalence was found among those remaining in manual occupations (and low educational group). ${ }^{23}$ Conversely, the upwardly mobile and those remaining in non-manual occupations and high education groups reported the least walking (during work and leisure) and greatest amount of sitting during the working day. ${ }^{23}$

Differences in physical activity ${ }^{24}$ and inactivity ${ }^{25}$ in leisure time between men from the British Regional Heart Study were greatest between those remaining in non-manual (highest prevalence of LTPA and lowest prevalence of leisure-time inactivity) and manual occupational groups; however, estimates for upwardly mobile men were similar to men stable in non-manual occupational groups. ${ }^{24}{ }^{25}$ Downwardly mobile women from the British Women's Heart and Health Study were less likely, and upwardly mobile more likely, to be inactive when compared with women stable in the same parental occupational group. ${ }^{26}$ Women from all other groups were also more likely to be inactive when compared with women remaining in non-manual occupations, with the greatest difference found in odds of inactivity for women remaining in the manual occupational group. ${ }^{27}$

More pronounced age and sex-adjusted differences in prevalence of sports and exercise were found across social mobility groups of the Scottish Health Survey 2003 when compared with those reported above. ${ }^{28}$ Further, in models with alternating adjustment for parental and adult SEP, upwardly mobile groups had a higher prevalence of LTPA than those stable in their SEP of origin but lower than those stable in their destination SEP, with the reverse direction found for the downwardly mobile (table 2). ${ }^{28}$ Findings from offspring of the Renfrew/Paisley Study ${ }^{29}$ suggest that the stable non-manual and upwardly mobile groups had lowest levels of daily physical activity (workrelated activity plus LTPA) (table 2). The authors report that these findings were due to manual workers performing more daily activities than non-manual workers and that exercise levels outside work were similar for manual and non-manual classes. ${ }^{29}$

Mean reported exercise hours in the West of Scotland Collaborative Study tended to be highest in the stable high and lowest in the stable low groups. ${ }^{30} 31 \mathrm{Male}^{35}$ and female ${ }^{36}$ US-based physicians who had experienced upward social mobility reported similar levels of LTPA to physicians with equally advantaged parents in terms of occupation class ${ }^{35}$ and education $^{36}$ though exercise prevalence was somewhat higher among the female physicians with two higher educated parents (table 2). ${ }^{36}$ Elsewhere, no associations were found between intergenerational educational mobility and a score based on estimated frequency, intensity and duration of exercise in the Cardiovascular Risk in Young Finns Study. ${ }^{32}$ In contrast, age and sex-adjusted findings from the Adolescent Health and Lifestyle Survey ${ }^{33}$ showed that upward mobility was associated with lower likelihood of leisure-time inactivity among 16-18-year-old Finns and that downward mobility was associated with higher likelihood (table 2). Upwardly mobile children of farmers and blue-collar workers had a lower risk of no LTPA than those stable in the same group while the downwardly mobile from upper white-collar and lower white-collar families had higher risk. ${ }^{33}$ Likewise, downwardly mobile Swedish men born in 1913 performed less exercise than men stable in high SEP. $^{34}$

Australian men and women aged 26-36 remaining in the highest and lowest educational groups between childhood and adulthood had the highest and lowest prevalence of LTPA, respectively, while socially mobile groups had levels in between these stable groups. ${ }^{37}$ Other findings from this cohort showed upwardly mobile men and women, and men stable in the high educational group, were more likely to increase LTPA between ages 9-15 and 26-36 than those stable in the low educational group. ${ }^{38}$ When compared with those always non-poor based on income from a 23-year-old Pelotas birth cohort, ${ }^{39}$ men and women who became poor adults and those who were always poor were both less likely to participate in LTPA (table 2).

\section{DISCUSSION}

\section{Main findings}

This systematic review included findings from 13 studies (reported in 17 publications). It found that intergenerational stability and mobility of SEP was consistently associated with LTPA in adulthood. Of 11 studies that examined intergenerational stability and upward and downward mobility of SEP, nine found similar patterns of association. These suggested that stable high socioeconomic groups reported the highest levels of LTPA and stable low socioeconomic groups the lowest, and that both socially mobile groups participated in LTPA at levels closer to the stable high SEP group. The other two of these 11 studies found no associations. In addition, there were no differences in prevalence of LTPA in the remaining two studies both of which compared physicians who were upwardly mobile with those who had stable high SEP.

\section{Explanation of findings}

The greatest differences in LTPA were between those groups stable in the same SEP and this supports an accumulation of additive effects hypothesis whereby continued exposure to a certain SEP in childhood and adulthood cumulatively alters probability of LTPA. ${ }^{11} 4142$ This is consistent with studies showing that those with low SEP in childhood and adulthood tend to have the worst health outcomes. ${ }^{12-16}{ }^{43-47}$ Upwardly mobile groups generally reported more LTPA than those remaining in low SEP of origin which may reflect an adoption of aspirational lifestyle of their destination socioeconomic group. ${ }^{9} 10$ This finding could also be partly due to upwardly mobile individuals working in more sedentary occupations and thus having more energy to participate in LTPA. ${ }^{23}$ The lower occupational physical activity of adults with higher SEP is also likely to explain the null or opposing findings of studies which included occupational physical activity as part of the outcome. ${ }^{23} 29$ Downwardly mobile groups tended to report levels of LTPA more similar to the stable high SEP group than the stable low SEP group which may suggest maintenance of health behaviours adopted in childhood. ${ }^{7} 904849$

This review's findings also suggest that transitioning to more advantaged SEP in adulthood may partially offset the influence of low childhood SEP on less LTPA in adulthood shown in previous reviews. ${ }^{7}{ }^{8}$ However, the relationship between SEP and 


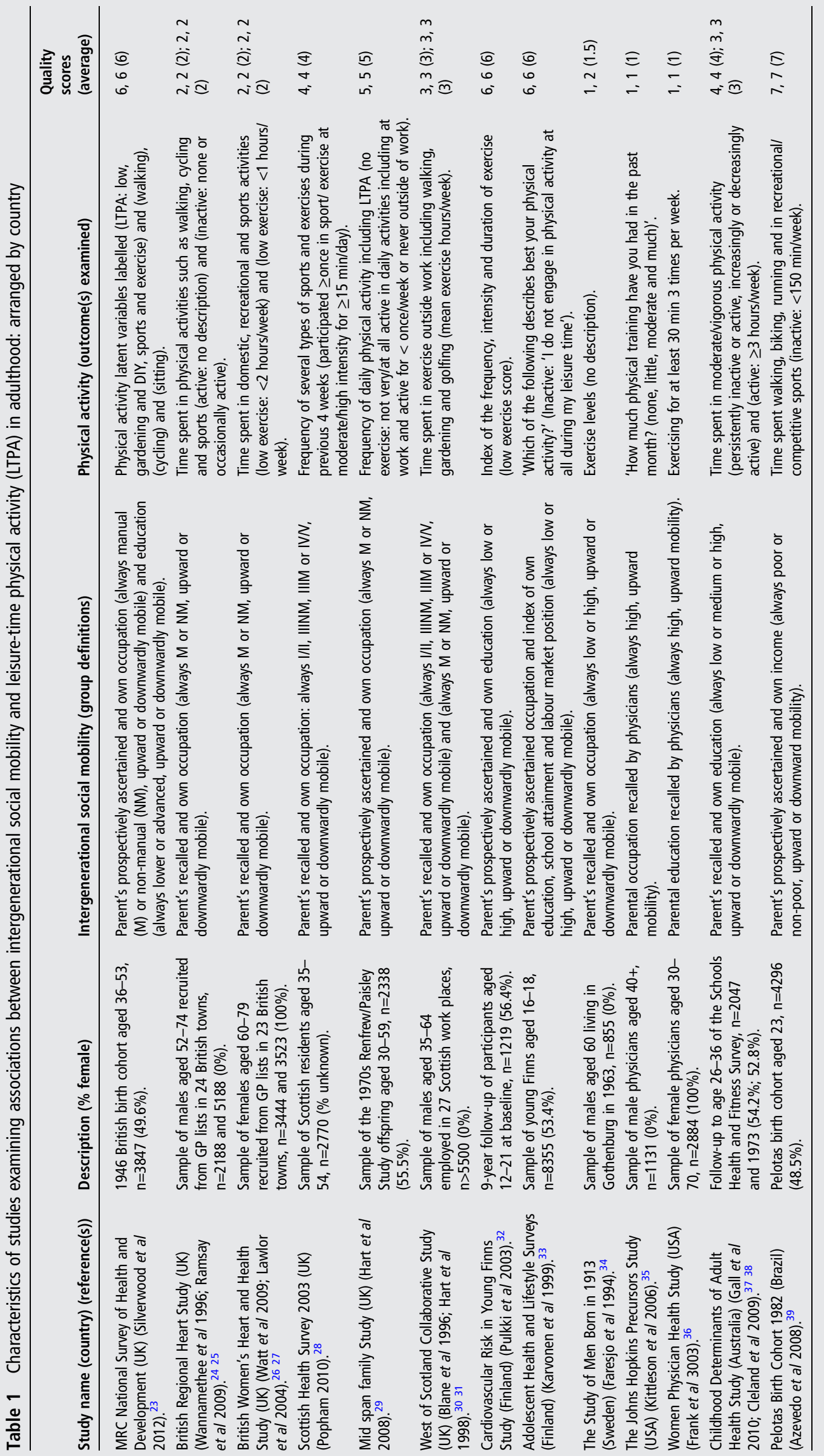




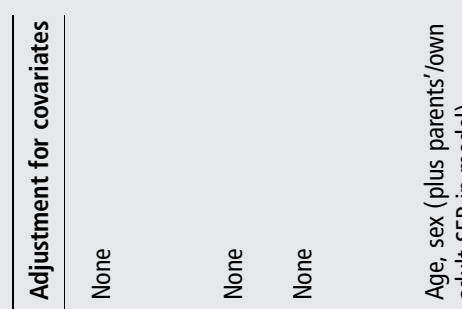

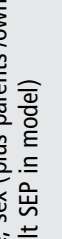

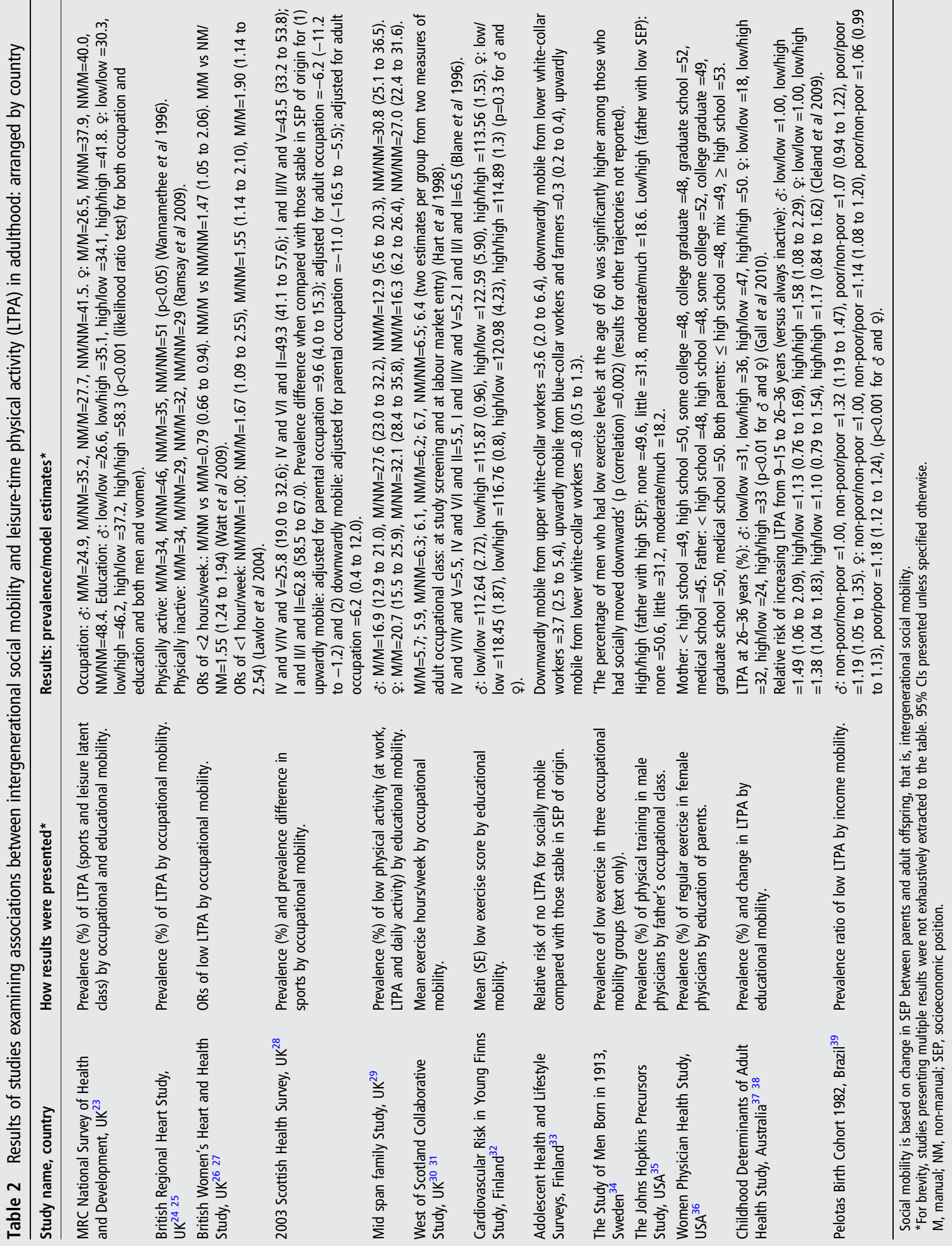


health of adults is influenced by processes operating during early life which can impact on their health (and related behaviours) and SEP. ${ }^{41} 46 \quad 50-55$ Moreover, childhood SEP may be more important for adult health in certain settings than others, for example, a study found father's education to be more important than own education in explaining differences in selfrated health in Eastern when compared with Western Europe. ${ }^{10}$

\section{Methodological considerations}

In assessing the published evidence it is important to consider methodological factors which may influence interpretation. An important limitation of most analyses identified was that they were unadjusted for potential confounders even though certain factors might influence social mobility and LTPA. Related to this, none of the studies described whether the upwardly socially mobile participated in LTPA more, and the downwardly mobile participated in LTPA less, than expected relative to the SEP group they joined which may have indicated selection effects and so helped differentiate genuine effects of social mobility from those due to confounding. ${ }^{51} 52$ In addition, studies tended not to empirically test whether social mobility or other life course models of association best fitted the data.

Most studies relied on recall of childhood SEP which could lead to misclassification due to recall errors and subsequently underestimate associations. ${ }^{56}$ Most studies also relied on binary classifications of childhood and adulthood SEP which may have removed some meaningful variation in patterns of SEP across life. Alternative measures of SEP were rarely considered, for example, household wealth may be an important indicator of SEP in older adults ${ }^{57}$ and it may also be useful to distinguish between types of education. ${ }^{58}$ Social mobility was limited to two time points in all studies; however, duration of exposure to different social positions across life may also influence findings. ${ }^{59}$ Further, all studies examined relative mobility without full consideration of contextual changes. In addition, associations with LTPA may change with age but this could not be examined as most studies included a single measure of LTPA. Lack of difference in LTPA between physicians from different socioeconomic backgrounds may suggest that adult SEP was more closely related to concurrent LTPA but could also reflect insufficient variation in childhood SEP.

All studies relied on participant reports to assess LTPA and although such methods are well suited to capturing LTPA, ${ }^{60} 61$ they can be subject to recall errors, particularly among older participants. ${ }^{62}$ Differential reporting of LTPA by SEP groups is also possible and could bias findings, for example, obesity tends to be more prevalent in lower SEP groups. ${ }^{13-15}$ and obese individuals have previously been found to be more likely to overestimate their levels of physical activity and energy expenditure. $^{63} 64$ Finally, most studies were in high-income countries and thus findings may not be generalisable to lowincome or middle-income settings.

\section{Strengths and limitations of the review}

Our systematic review has several important strengths that include the use of a protocol, following of established guidelines, searching of multiple databases and reference lists, and assessment of search results and included studies by pairs of independently working reviewers which helps prevent errors in screening and data extraction. Despite our efforts to locate all published studies, a wider search may have identified additional studies (eg, of economics journals databases and non-English language studies). In addition, we did not search for unpublished studies and we could not formally test for evidence of publication bias as we did not perform a meta-analysis. However, potential publication bias may have been minimised by inclusion of all studies even where associations of interest were not the primary aim.

\section{Implications of findings}

A better understanding of the mechanisms through which socioeconomic circumstances might influence LTPA is required. Studies with repeat assessments of SEP could test alternate hypotheses relating life course SEP to LTPA. ${ }^{14} 164265$ and studies with repeat assessments of LTPA could examine whether associations vary by age. Studies with repeat measures could also use within-person designs as a means of accounting for baseline confounders ${ }^{66}$ and attention should be paid to factors which contextualise SEP such as family and labour market experiences. ${ }^{46}$ Moreover, alternative study designs which offer natural confounder adjustment such as twin studies ${ }^{67} 68$ could help identify the relative importance of early life and adult socioeconomic circumstances for later LTPA, which may help inform appropriate timing of interventions.

The findings of this review suggest that policies and interventions aimed at minimising exposure to socioeconomic adversity ${ }^{69-73}$ could lead to increases in LTPA. These should focus on reducing socioeconomic adversity rather than changing class structure as the latter would result in some people experiencing downward mobility. To this end, improving early life conditions and socioeconomic circumstances may benefit socioeconomic potential and subsequent LTPA. ${ }^{7} 8$ 69-78

\section{CONCLUSIONS}

This systematic review of intergenerational social mobility associations with adult LTPA included 13 studies and found that those in stable high socioeconomic groups reported the highest levels of participation in LTPA and those in stable low socioeconomic groups the lowest, and that socially mobile groups participated in LTPA at levels between these stable groups. Thus, policies which aim to minimise exposure to socioeconomic adversity may result in improved LTPA levels.

\section{What is already known on this subject}

- Recent systematic reviews have reported associations between lower socioeconomic position (SEP) in childhood and less leisure-time physical activity (LTPA) in adulthood.

- The association between intergenerational social mobility and LTPA in adulthood has not previously been systematically reviewed.

\section{What this study adds}

- This is the first systematic review of published evidence on the association between intergenerational social mobility and adult LTPA.

- Cumulative exposure to higher SEP in childhood and adulthood was associated with higher LTPA among adults from different countries.

- Policies which aim to minimise exposure to socioeconomic adversity at any point in life may have the potential to improve LTPA status in adulthood. 
Twitter Follow Theodore Cosco @tdcosco

Contributors $A E, R H, D B$ and $R C o$ designed the review. $A E$ registered the protocol with PROSPERO. AE, RCa, TDC, RPGH, SGM and RW carried out independent screening, data extraction and quality assessment. AE drafted the manuscript and all authors contributed to its critical revision and approved its final version.

Funding $A E, R H, S G M, R W$ and RCo are supported by the Medical Research Council (MRC) (programme codes MC_UU_12019/1, MC_UU_12019/2 and MC_UU_12019/4). SGM is also supported by MRC grant MR/L010399/1. TDC is supported by a Canadian Institutes of Health Research Postdoctoral Fellowship (MFE-146676).

Disclaimer The funders had no role in the design of the study or the writing of the manuscript and played no part in the decision to submit it for publication.

Competing interests None declared.

Provenance and peer review Not commissioned; externally peer reviewed.

Open Access This is an Open Access article distributed in accordance with the terms of the Creative Commons Attribution (CC BY 4.0) license, which permits others to distribute, remix, adapt and build upon this work, for commercial use, provided the original work is properly cited. See: http://creativecommons.org/licenses/ by/4.0/

\section{REFERENCES}

1 Warburton DE, Nicol CW, Bredin SS. Health benefits of physical activity: the evidence. CMAJ 2006;174:801-9.

2 Lee IM, Shiroma EJ, Lobelo $F$, et al. Effect of physical inactivity on major non-communicable diseases worldwide: an analysis of burden of disease and life expectancy. Lancet 2012;380:219-29.

3 World Health Organization. Global recommendations on physical activity for health. Geneva, WHO Press, 2010. ISBN: 9789241599979.

4 Stalsberg R, Pedersen AV. Effects of socioeconomic status on the physical activity in adolescents: a systematic review of the evidence. Scand J Med Sci Sports 2010;20:368-83.

5 Gidlow $C$, Johnston $\mathrm{LH}$, Crone $\mathrm{D}$, et al. A systematic review of the relationship between socio-economic position and physical activity. Health Educ J 2006;65:338-67.

6 Beenackers MA, Kamphuis CB, Giskes K, et al. Socioeconomic inequalities in occupational, leisure-time, and transport related physical activity among European adults: a systematic review. Int J Behav Nutr Phys Act 2012;9:116.

7 Elhakeem A, Cooper R, Bann D, et al. Childhood socioeconomic position and adult leisure-time physical activity: a systematic review. Int J Behav Nutr Phys Act 2015;12:92.

8 Juneau CE, Benmarhnia T, Poulin AA, et al. Socioeconomic position during childhood and physical activity during adulthood: a systematic review. Int J Public Health 2015:60:799-813.

9 Missinne S, Daenekindt S, Bracke P. The social gradient in preventive healthcare use: what can we learn from socially mobile individuals? Sociol Health IIIn 2015;37:823-38

10 Monden CW, de Graaf ND. The importance of father's and own education for self-assessed health across Europe: an East-West divide? Sociol Health IIIn 2013:35:977-92

11 Kuh D, Ben-Shlomo Y, Lynch J, et al. Life course epidemiology. J Epidemiol Community Health 2003;57:778-83.

12 Poulton R, Caspi A, Milne BJ, et al. Association between children's experience of socioeconomic disadvantage and adult health: a life-course study. Lancet 2002:360:1640-5.

13 Heraclides A, Brunner E. Social mobility and social accumulation across the life course in relation to adult overweight and obesity: the Whitehall II study. J Epidemiol Community Health 2010;64:714-19.

14 Pollitt RA, Rose KM, Kaufman JS. Evaluating the evidence for models of life course socioeconomic factors and cardiovascular outcomes: a systematic review. BMC Public Health 2005;5:7.

15 Parsons TJ, Power C, Logan S, et al. Childhood predictors of adult obesity: a systematic review. Int J Obes Relat Metab Disord 1999:23(Suppl 8):S1-107.

16 Murray ET, Mishra GD, Kuh D, et al. Life course models of socioeconomic position and cardiovascular risk factors: 1946 birth cohort. Ann Epidemiol 2011;21:589-97.

17 Moher D, Liberati A, Tetzlaff J, et al. Preferred reporting items for systematic reviews and meta-analyses: the PRISMA statement. BMJ 2009;339:b2535.

18 Krieger N, Williams DR, Moss NE. Measuring social class in US public health research: concepts, methodologies, and guidelines. Annu Rev Public Health 1997:18:341-78

19 Galobardes B, Shaw M, Lawlor DA, et al. Indicators of socioeconomic position (part 1). J Epidemiol Community Health 2006;60:7-12.

20 Howley ET. Type of activity: resistance, aerobic and leisure versus occupational physical activity. Med Sci Sports Exerc 2001;33(6 Suppl):S364-9.
21 Elhakeem A, Cooper R, Bann D, et al. Childhood socioeconomic position and adult leisure-time physical activity: a systematic review protocol. Syst Rev 2014;3:141.

22 The Newcastle-Ottawa Quality Assessment Scale. http://www.ohri.ca/programs/ clinical epidemiology/nosgen.pdf (accessed 20 Feb 2015).

23 Silverwood RJ, Pierce $M$, Nitsch $D$, et al. Is intergenerational social mobility related to the type and amount of physical activity in mid-adulthood? Results from the 1946 British birth cohort study. Ann Epidemiol 2012;22:487-98.

24 Wannamethee SG, Whincup PH, Shaper G, et al. Influence of fathers' social class on cardiovascular disease in middle-aged men. Lancet 1996;348:1259-63.

25 Ramsay SE, Whincup PH, Morris RW, et al. Are childhood socio-economic circumstances related to coronary heart disease risk? Findings from a population-based study of older men. Int J Epidemiol 2007:36:560-6.

26 Watt HC, Carson C, Lawlor DA, et al. Influence of life course socioeconomic position on older women's health behaviors: findings from the British Women's Heart and Health Study. Am J Public Health 2009:99:320-7.

27 Lawlor DA, Smith GD, Ebrahim S. Association between childhood socioeconomic status and coronary heart disease risk among postmenopausal women: findings from the British Women's Heart and Health Study. Am J Public Health 2004;94:1386-92.

28 Popham F. Intergenerational social class stability and mobility are associated with large absolute differences in adult participation in sport and exercise. $\mathrm{Br} J$ Sports Med 2010;44:1184-5.

29 Hart C, McConnachie A, Upton M, et al. Risk factors in the Midspan family study by social class in childhood and adulthood. Int J Epidemiol 2008;37:604-14.

30 Blane D, Hart CL, Smith GD, et al. Association of cardiovascular disease risk factors with socioeconomic position during childhood and during adulthood. BMJ 1996;313:1434-8.

31 Hart CL, Smith GD, Blane D. Social mobility and 21 year mortality in a cohort of Scottish men. Soc Sci Med 1998;47:1121-30

32 Pulkki L, Kivimäki M, Elovainio $M$, et al. Contribution of socioeconomic status to the association between hostility and cardiovascular risk behaviors: a prospective cohort study. Am J Epidemiol 2003;158:736-42.

33 Karvonen S, Rimpelä AH, Rimpelä MK. Social mobility and health related behaviours in young people. J Epidemiol Community Health 1999;53:211-17.

34 Faresjö T, Svärdsudd K, Tibblin G. Social mobility and health in a prospective study of middle-aged men. Scand J Soc Med 1994;22:86-9.

35 Kittleson MM, Meoni LA, Wang NY, et al. Association of childhood socioeconomic status with subsequent coronary heart disease in physicians. Arch Intern Med 2006:166:2356-61.

36 Frank E, Elon L, Hogue C. Transgenerational persistence of education as a health risk: findings from the Women Physicians' Health Study. J Womens Health (Larchmt) 2003:12:505-12.

37 Gall SL, Abbott-Chapman J, Patton GC, et al. Intergenerational educational mobility is associated with cardiovascular disease risk behaviours in a cohort of young Australian adults: The Childhood Determinants of Adult Health (CDAH) Study. BMC Public Health 2010;10:55.

38 Cleland VJ, Ball K, Magnussen C, et al. Socioeconomic position and the tracking of physical activity and cardiorespiratory fitness from childhood to adulthood. Am J Epidemiol 2009;170:1069-77

39 Azevedo MR, Horta BL, Gigante DP, et al. [Factors associated to leisure-time sedentary lifestyle in adults of 1982 birth cohort, Pelotas, Southern Brazil]. Rev Saude Publica 2008;42(Suppl 2):70-7.

40 Silverwood RJ, Nitsch D, Pierce M, et al. Characterizing longitudinal patterns of physical activity in mid-adulthood using latent class analysis: results from a prospective cohort study. Am J Epidemiol 2011;174:1406-15.

41 Kuh D, Power C, Blane D, et al. Socioeconomic pathways between childhood and adult health. In: Kuh D, Ben-Shlomo Y, eds. A life course approach to chronic disease epidemiology. 2nd edn. New York: Oxford University Press, 2004:371-98.

42 Juneau $C E$, Sullivan $A$, Dodgeon $B$, et al. Social class across the life course and physical activity at age 34 years in the 1970 British birth cohort. Ann Epidemiol 2014;24:641-7,647.e1.

43 Lynch JW, Kaplan GA, Cohen RD, et al. Childhood and adult socioeconomic status as predictors of mortality in Finland. Lancet 1994;343:524-7.

44 Bartley M. Plewis I. Increasing social mobility: an effective policy to reduce health inequalities. J R Stat Soc 2007;170:469-81.

45 Stringhini $S$, Batty GD, Bovet $P$, et al. Association of lifecourse socioeconomic status with chronic inflammation and type 2 diabetes risk: the Whitehall II prospective cohort study. PLoS Med 2013:10:e1001479.

46 Corna LM. A life course perspective on socioeconomic inequalities in health: a critical review of conceptual frameworks. Adv Life Course Res 2013:18:150-9.

47 Brunner E, Shipley MJ, Blane D, et al. When does cardiovascular risk start? Past and present socioeconomic circumstances and risk factors in adulthood. J Epidemiol Community Health 1999;53:757-64.

48 Telama R, Yang $X$, Leskinen $E$, et al. Tracking of physical activity from early childhood through youth into adulthood. Med Sci Sports Exerc 2014;46:955-62.

49 Smith L, Gardner B, Aggio D, et al. Association between participation in outdoor play and sport at 10 years old with physical activity in adulthood. Prev Med 2015;74:31-5. 
50 Kröger H, Pakpahan E, Hoffmann R. What causes health inequality? A systematic review on the relative importance of social causation and health selection. Eur J Public Health 2015;25:951-60.

51 Blane D, Davey Smith G, Bartley M. Social selection: what does it contribute to social class differences in health? Sociol Health IIIn 1993;15:1-15.

52 Bartley M, Plewis I. Does health-selective mobility account for socioeconomic differences in health? Evidence from England and Wales, 1971 to 1991. J Health Soc Behav 1997:38:376-86.

53 Manor O, Matthews S, Power C. Health selection: the role of inter- and intra-generational mobility on social inequalities in health. Soc Sci Med 2003;57:2217-27.

54 Sweeting $\mathrm{H}$, Green $\mathrm{M}$, Benzeval $\mathrm{M}$, et al. The emergence of health inequalities in early adulthood: evidence on timing and mechanisms from a West of Scotland cohort. BMC Public Health 2016;16:41.

55 Foverskov E, Holm A. Socioeconomic inequality in health in the British household panel: tests of the social causation, health selection and the indirect selection hypothesis using dynamic fixed effects panel models. Soc Sci Med 2016;150:172-83.

56 Batty GD, Lawlor DA, Macintyre S, et al. Accuracy of adults' recall of childhood social class: findings from the Aberdeen children of the 1950s study. J Epidemiol Community Health 2005;59:898-903.

57 Demakakos P, Biddulph JP, Bobak M, et al. Wealth and mortality at older ages: a prospective cohort study. J Epidemiol Community Health 2016;70:346-53.

58 Bann D, Hamer M, Parsons S, et al. Does an elite education benefit health? Findings from the 1970 British Cohort Study. Int J Epidemio/ Epub ahead of print: 10 May 2016. doi: 10.1093/ije/dyw045

59 Power C, Manor 0, Matthews S. The duration and timing of exposure: effects of socioeconomic environment on adult health. Am J Public Health 1999;89:1059-65

60 Troiano RP, McClain JJ, Brychta RJ, et al. Evolution of accelerometer methods for physical activity research. Br J Sports Med 2014;48:1019-23.

61 Kelly P, Fitzsimons C, Baker G. Should we reframe how we think about physical activity and sedentary behaviour measurement? Validity and reliability reconsidered. Int J Behav Nutr Phys Act 2016;13:32.

62 Schrack JA, Cooper R, Koster A, et al. Assessing daily physical activity in older adults: unraveling the complexity of monitors, measures, and methods. J Gerontol A Biol Sci Med Sci 2016;71:1039-48.

63 Lichtman SW, Pisarska K, Berman ER, et al. Discrepancy between self-reported and actual caloric intake and exercise in obese subjects. $N$ Engl J Med 1992;327:1893-8.
64 Warner ET, Wolin KY, Duncan DT, et al. Differential accuracy of physical activity self-report by body mass index. Am J Health Behav 2012;36:168-78.

65 Jones R, Hardy R, Sattar N, et al. Novel coronary heart disease risk factors at 60 64 years and life course socioeconomic position: the 1946 British birth cohort. Atherosclerosis 2015;238:70-6.

66 Gunasekara FI, Richardson K, Carter K, et al. Fixed effects analysis of repeated measures data. Int J Epidemiol 2014;43:264-9.

67 Osler M, McGue M, Christensen K. Socioeconomic position and twins' health: a life-course analysis of 1266 pairs of middle-aged Danish twins. Int J Epidemiol 2007;36:77-83.

68 Krieger N, Chen JT, Coull BA, et al. Lifetime socioeconomic position and twins' health: an analysis of 308 pairs of United States women twins. PLoS Med 2005;2:e162.

69 HM Government. Opening Doors, Breaking Barriers: A Strategy for Social Mobility. 2011. https://www.gov.uk/government/uploads/system/uploads/attachment_data/file/ 61964/opening-doors-breaking-barriers.pdf (accessed 25Jun 2016).

70 Social Mobility Commission. The childhood origins of social mobility: socio-economic inequalities and changing opportunities. 2016. https://www.gov.uk/government/ uploads/system/uploads/attachment_data/file/528315/The_childhood_origins_of_ social_mobility.pdf (accessed 25 Jun 2016).

71 Causa 0, Johansson $\AA$. Intergenerational social mobility in OECD countries. OECD J: Econ Stud 2010;1:1-44.

72 Chetty R, Friedman JN, Rockoff JE. Measuring the Impacts of Teachers II: teacher value-added and student outcomes in adulthood. Am Econ Rev 2014; 104:2633-79.

73 Chetty R, Hendren N, Kline K, et al. Where is the land of opportunity? The geography of intergenerational mobility in the United States. $Q J$ Econ 2014;129:1553-623.

74 Sacker $A$, Kelly $Y$, lacovou $M$, et al. Breast feeding and intergenerational social mobility: what are the mechanisms? Arch Dis Child 2013;98:666-71.

75 Pinto Pereira SM, Li L, Power C. Early-life predictors of leisure-time physical inactivity in midadulthood: findings from a prospective British birth cohort. Am J Epidemiol 2014;180:1098-108.

76 Pinto Pereira SM, Li L, Power C. Early life factors and adult leisure time physica inactivity stability and change. Med Sci Sports Exerc 2015;47:1841-8.

77 Elhakeem A, Cooper R, Bann D, et al. Birth weight, school sports ability and adulthood leisure-time physical activity. Med Sci Sports Exerc Epub ahead of print: 30 Aug 2016. doi: 10.1249/MSS.0000000000001077

78 Kuh DJ, Cooper C. Physical activity at 36 years: patterns and childhood predictors in a longitudinal study. J Epidemiol Community Health 1992:46:114-19. 